\title{
RANKL/RANK pathway abrogates cetuximab sensitivity in gastric cancer cells via activation of EGFR and c-Src
}

This article was published in the following Dove Press journal:

OncoTargets and Therapy

5 January 2017

Number of times this article has been viewed

\author{
Xiaomeng Zhang ${ }^{1,3}$ \\ Yongxi Song ${ }^{2}$ \\ $\mathrm{Na}$ Songl,3 \\ Ye Zhang ${ }^{1,3}$ \\ Lingyun Zhangl,3 \\ Yan Wang ${ }^{1,3}$ \\ Zhenning Wang ${ }^{2}$ \\ Xiujuan $\mathrm{Qu}^{1,3}$ \\ Yunpeng Liu ${ }^{1,3}$ \\ 'Department of Medical Oncology, \\ 2Department of Surgical Oncology \\ and General Surgery, ${ }^{3}$ Key Laboratory \\ of Anticancer Drugs and Biotherapy of \\ Liaoning Province, The First Hospital \\ of China Medical University, Shenyang, \\ People's Republic of China
}

Correspondence: Xiujuan Qu;

Yunpeng Liu

Department of Medical Oncology, Key Laboratory of Anticancer Drugs and Biotherapy of Liaoning Province, The First Hospital of China Medical University, I 55 North Nanjing Street, Heping District, Shenyang I I000 I,

People's Republic of China

Tel +86 2483282312

Fax +86248328 2543

Email xiujuanqu@yahoo.com; cmuliuyunpeng@hotmail.com
Abstract: Overexpression of EGFR is commonly seen in gastric cancer (GC). However, patients with GC show resistance to anti-EGFR treatments. $R A S$ mutations are rare in GC and cannot explain de novo resistance to EGFR treatments. Therefore, it is particularly important to explore the mechanisms of resistance to anti-EGFR treatments. The RANKL activates the EGFR pathway in osteoclasts, and the RANK is expressed in gastric carcinoma. Whether the RANKL/RANK pathway has an effect on the EGFR pathway in GC remains unknown. Expressions of EGFR and RANK in GC tissues were detected using immunohistochemical staining. Nineteen patients (28\%) showed high-level RANKL expression, and 33 patients $(48 \%)$ showed high-level RANK expression. There was a positive correlation between expression of EGFR and RANK $(P<0.001)$. In an in vitro study, RANKL induced activation of the EGFR pathway and further abrogated cetuximab sensitivity in GC cells. Knockdown of RANK or use of the RANKL inhibitor enhanced cetuximab effect by decreasing RANKL-induced EGFR activation. Furthermore, we showed that c-SRC mediated the EGFR activation induced by the RANKL/RANK pathway and that c-SRC inhibitor reversed the suppression of RANKL on the effect of cetuximab. In conclusion, our results suggest that in GC cells, the RANKL/RANK pathway activates the EGFR pathway and thereby causes resistance to anti-EGFR treatments. Keywords: RANKL/RANK, EGFR, cetuximab, c-Src, gastric cancer

\section{Introduction}

Gastric cancer (GC) is the fourth commonly diagnosed cancer and the second most frequent cause of cancer death worldwide. ${ }^{1}$ Although comprehensive therapies have improved the response rate, patients with advanced GC continue to have a poor prognosis. ${ }^{2}$ Overexpression of EGFR is associated with poor prognosis in GCs, ${ }^{3,4}$ yet clinical studies using anti-EGFR agents in the general population have failed to show a significant improvement. ${ }^{5,6}$ Therefore, it is necessary to explore the resistance mechanisms to anti-EGFR agents in these patients. Mutations in RAS oncogenes serve as the major predictive biomarker of anti-EGFR treatments in colorectal cancer. However, the mutation rate for $R A S$ is $\sim 5 \%$ in GC. ${ }^{5}$ Therefore, searching for alternative resistance mechanisms to anti-EGFR agents is of critical importance in GC patients.

One crucial resistance mechanism to anti-EGFR agents is aberrant activation of EGFR by alternative pathways, such as c-Met and IGF-IR, ${ }^{7,8}$ that activate many of the same downstream signaling pathways as EGFR. RANKL is involved in osteoclast differentiation and induces EGFR activation. ${ }^{9}$ RANKL is a member of the tumor necrosis factor family of cytokines, and its receptor RANK was previously found to promote osteoclast precursor maturation. Expression of RANK was found in several 
solid tumors such as breast, prostate and hepatocellular carcinomas. ${ }^{10-12}$ Recently, the RANKL/RANK pathway was shown to promote cancer cell migration by stimulating AKT and ERK, which are regarded as the most important downstream mediators of EGFR signaling. ${ }^{13}$ Therefore, we speculated that the RANKL/RANK pathway might prove vital for EGFR activation in GC cells.

In this study, we explored the correlation between RANKL and EGFR expression, and the role of the RANKL/ RANK pathway on the activation of EGFR signaling and resistance to anti-EGFR agents in GC. Our results might suggest a novel mechanism of resistance to anti-EGFR therapies in the treatment of $\mathrm{GC}$.

\section{Materials and methods Reagents}

Cetuximab was purchased from EMD Millipore (Billerica, MA, USA). Recombinant sRANKL and rOPG was purchased from CytoLab/PeproTech Asia (USA). PP2 was obtained from Sigma-Aldrich Co. (St Louis, MO, USA). Dasatinib was obtained from Selleck Chemicals (Houston, TX, USA). S-P immunohistochemical kit and 3,3'diaminobenzidine tetrahydrochloride kit were obtained from Maixin Bio (Fuzhou Maixin Biological Technology Ltd., Fujian, People's Republic of China).

\section{Cell cultures}

GC cells such as SGC-7901, MGC-803, BGC-823, MKN-45, and KATO-III and colon cancer cells such as Caco-2 were obtained from The Cell Bank of Type Culture Collection of the Chinese Academy of Sciences (People's Republic of China). Cells were cultured in Roswell Park Memorial Institute 1640 medium (Thermo Fisher Scientific, Waltham, MA, USA) containing 10\% fetal bovine serum (FBS), penicillin $(100 \mathrm{U} / \mathrm{mL})$, and streptomycin $(100 \mathrm{mg} / \mathrm{mL})$ in an atmosphere of $95 \%$ air and $5 \% \mathrm{CO}_{2}$ at $37^{\circ} \mathrm{C}$.

\section{Patients and tissue samples}

Specimens of gastric adenocarcinoma tissue were collected from 68 patients who underwent surgical resection at the First Affiliated Hospital of China Medical University. None of them received preoperative radiotherapy, chemotherapy or immunotherapy. Age, sex, pathological tumor-nodemetastasis (pTNM) stage, and Lauren grade were evaluated following medical charts and pathology records. pTNM stage was examined according to the seventh edition of $A J C C$ Cancer Staging Manual. Lauren grade was according to the classification of World Health Organization. The First Affiliation Hospital of China Medical University Ethical
Committee approved this study, and consent was not needed due to the retrospective nature of the research.

\section{Cell viability assay}

The effect of cell proliferation was measured using an MTT assay. Cells were seeded at 3,000-5,000/well in 96-well plates and incubated at $37^{\circ} \mathrm{C}$ for 24 hours in $10 \% \mathrm{FBS}$ medium. After pretreatment with dasatinib for 1 hour or not, the cells were incubated with the indicated doses of cetuximab, RANKL or a combination of them for 48 hours. MTT assay was performed as previously described. ${ }^{14}$

\section{Colony-forming assay}

Cells were seeded at 300 cells (SGC-7901, MGC-803), 500 cells (Caco-2) per well in 12-well plates and exposed to $10 \mu \mathrm{g} / \mathrm{mL}$ cetuximab, $1 \mu \mathrm{g} / \mathrm{mL}$ RANKL or a combination of them treatment after plating for 24 hours in 10\% FBS medium. Then followed by staining with Wright-Giemsa after 14 days, the number of colonies was counted.

\section{Western blot}

Western blot was performed as previously described. ${ }^{14}$ The following antibodies were used: Anti-RANK antibody was obtained from Bethyl Laboratories, Inc. (Montgomery, TX, USA). Anti-actin antibody was produced by Santa Cruz Biotechnology Inc. (Dallas, TX, USA). AntiEGFR, anti-phospho-EGFR (Tyr1068), anti-AKT, antip-AKT (Ser473), anti-ERK1/2, anti-p-ERK1/2 (Thr202/ Tyr204), anti-c-Src, and anti-p-c-Src (Y416) antibodies were obtained from Cell Signaling Technology (Danvers, MA, USA). Secondary goat anti-rabbit and goat anti-mouse antibodies were purchased from Santa Cruz Biotechnology Inc.

\section{Immunohistochemistry}

Formalin-fixed, paraffin-embedded primary gastric carcinoma tissues were cut into $3 \mathrm{~mm}$ sections. The method of immunohistochemistry (IHC) is discussed in our previous study. ${ }^{15}$ Immunohistochemical staining was performed using the following antibodies: anti-RANK antibody from RD Company and anti-EGFR antibody from Santa Cruz Biotechnology Inc. For the evaluation of immunohistochemical, sections were observed through microscopic examining $(\times 20$ and $\times 40)$ assigned by two independent pathologists. From each section, five visual fields were randomly selected, and the score for each visual field was based on the percentage of positive cells and the staining intensity. For the percentage of positive cells, $<10 \%, 10 \%-25 \%, 26 \%-50 \%$, $51 \%-75 \%$, and $>76 \%$ were recorded as $0,1,2,3$, and 4 
points, respectively. A score $>2$ was classified as a high-level expression and $0-2$ as a low-level expression.

\section{Small interfering RNA transfections}

RANK small interfering RNA (siRNA) was obtained form RiboBio Co., Ltd. (Guangzhou, People's Republic of China). RANK siRNA was synthesized: 5'-GGCAGGUGAU GAACUUCAA-3'. The control siRNA was AATTCTC CGAACGTGT CACGT. Cells were transfected with siRNAs using Lipofectamine 2000 (Thermo Fisher Scientific) according to the manufacturer's instructions. After 48 hours of transient transfection, the cells were analyzed by Western blot to verify gene-silencing efficiency.

\section{Statistic analysis}

Data were confirmed in three independent experiments and were expressed as mean \pm standard deviation. Differences between groups were compared using Student's $t$-test. The correlation between EGFR and RANK expression was assessed using Spearman's rank correlation for continuous variables. $P<0.05$ was considered statistically significant. Statistic analysis was carried out using SPSS 21.0 software package (IBM Corporation, Armonk, NY, USA).

\section{Results}

\section{Expression of EGFR shows a positive correlation with RANK expression in GC patients}

A total of 68 primary GC samples were obtained, and the expression of EGFR and RANK was determined by IHC.
High-level EGFR expression was detected in 19 patients (28\%), and 33 patients (48\%) showed high-level RANK expression (Figure 1A). Correlations between expression of EGFR and RANK and clinical characteristics of primary GC patients are given in Table 1. Importantly, there was a significant positive correlation between the expression of EGFR and RANK ( $P<0.001$; Table 2). Furthermore, we characterized a panel of five human GC cell lines (SGC-7901, MGC-803, BGC-823, MKN-45, and KATO-III) by Western blot to detect the cellular expression levels of EGFR and RANK. The results showed that EGFR and RANK expression was observed in most of the cell lines (Figure 1B).

Taken together, our results demonstrated that EGFR expression was positively correlated with RANK expression, suggesting an association between the RANKL/RANK pathway and EGFR signaling in GC.

\section{Stimulation with RANKL activates EGFR and downstream pathways in GC cells}

We continued to investigate the role of RANKL/RANK pathway in the EGFR pathway in vitro. We used SGC-7901 and MGC-803, which were frequently used in the People's Republic of China as RAS wild-type GC cell lines. Following stimulation with RANKL, we observed phosphorylation of EGFR, AKT, and ERK, indicating that they were transiently activated at different time points in both cell lines (Figure 2A).

To observe the effect of RANKL on cell proliferation, we treated the two cell lines with different concentrations of RANKL for 48 hours. An MTT assay revealed that RANKL
A
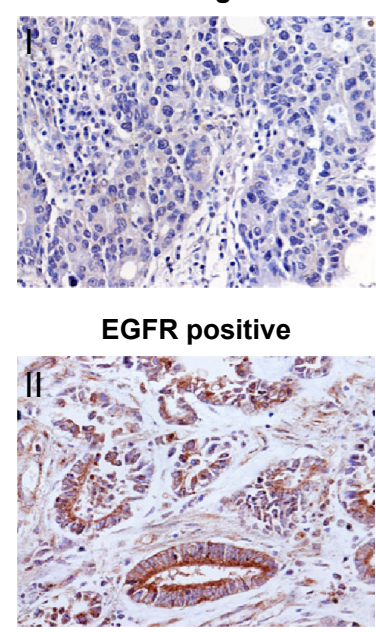

RANKnegative

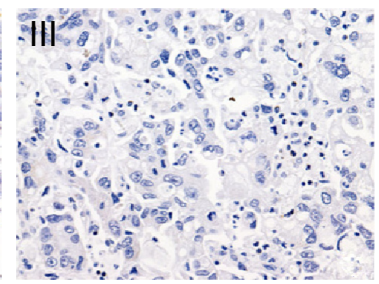

RANKpositive

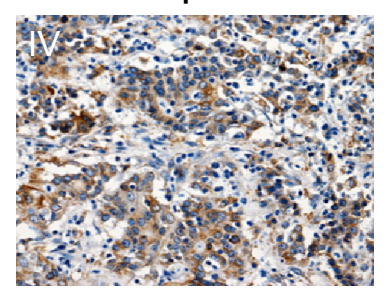

B

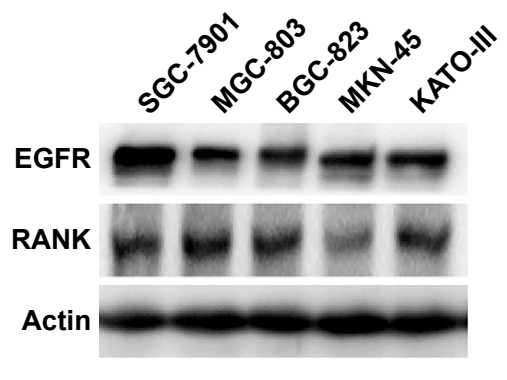

Figure I The expression of RANKL in GC tissues.

Notes: (A) (I and III) EGFR and RANKnegative staining and (II and IV) EGFR and positive staining, respectively (in brown). Magnification $\times 400$. (B) The expression of EGFR and RANK in SGC-790I, MGC-803, BGC-823, MKN-45 and KATO-III was detected by Western blot, using actin as a loading control.

Abbreviation: GC, gastric cancer. 
Table I Correlation between the expression of EGFR or RANK and the clinicopathological factors in primary GC patients

\begin{tabular}{|c|c|c|c|c|c|c|c|}
\hline \multirow[t]{2}{*}{ Characteristics } & \multirow[t]{2}{*}{ Cases } & \multicolumn{3}{|l|}{ EGFR } & \multicolumn{3}{|l|}{ RANK } \\
\hline & & Low (\%) & High (\%) & $P$-value & Low (\%) & High (\%) & $P$-value \\
\hline \multirow[t]{3}{*}{ Sex } & & & & 0.903 & & & 0.118 \\
\hline & Male & $38(72)$ & $15(28)$ & & $25(47)$ & $28(53)$ & \\
\hline & Female & II (73) & $4(27)$ & & $10(67)$ & $5(33)$ & \\
\hline \multirow[t]{3}{*}{ Age (years) } & & & & 0.907 & & & 0.335 \\
\hline & $<60$ & $24(73)$ & $9(27)$ & & $19(58)$ & $14(42)$ & \\
\hline & $\geq 60$ & 25 (7I) & $10(29)$ & & $16(46)$ & $19(54)$ & \\
\hline \multirow[t]{3}{*}{ pTNM stage } & & & & $0.019 *$ & & & $0.017^{*}$ \\
\hline & $I+I I$ & $16(94)$ & I (6) & & $13(76)$ & $4(24)$ & \\
\hline & III & $33(65)$ & $18(35)$ & & $22(43)$ & $29(57)$ & \\
\hline \multirow[t]{3}{*}{ T stage } & & & & $0.046^{*}$ & & & 0.093 \\
\hline & TI-2 & $9(100)$ & $0(0)$ & & 7 (78) & $2(22)$ & \\
\hline & T3-4 & $40(68)$ & $19(32)$ & & $28(48)$ & $31(52)$ & \\
\hline \multirow[t]{3}{*}{$N$ stage } & & & & $0.013^{*}$ & & & 0.136 \\
\hline & No & $17(94)$ & I (6) & & $12(67)$ & $6(33)$ & \\
\hline & $\mathrm{NI}-3$ & $32(64)$ & $18(36)$ & & $23(46)$ & $27(54)$ & \\
\hline \multirow[t]{4}{*}{ Lauren grade } & & & & 0.545 & & & 0.693 \\
\hline & Intestinal & $18(67)$ & $9(33)$ & & $12(44)$ & I5 (56) & \\
\hline & Diffuse & $17(77)$ & $5(23)$ & & $14(64)$ & $8(36)$ & \\
\hline & Mixed & $14(74)$ & $5(26)$ & & $9(47)$ & $10(53)$ & \\
\hline
\end{tabular}

Note: $* p<0.05$

Abbreviations: GC, gastric cancer; pTNM, pathological tumor-node-metastasis.

exhibited no distinct effect on cell proliferation at the concentration of $0.1 \mu \mathrm{g} / \mathrm{mL}$ or $1 \mu \mathrm{g} / \mathrm{mL}$ (Figure $2 \mathrm{~B}$ ).

\section{RANKL decreases sensitivity to cetuximab in GC cells}

Because RANKL activated the EGFR pathway, we speculated that the RANKL/RANK pathway could affect sensitivity to cetuximab.

First, we examined the proliferation of cells treated with cetuximab. Both cells exhibited mild growth inhibition after addition of cetuximab $(21.7 \% \pm 4.7 \%$ in SGC-7901 and $16.8 \% \pm 2.3 \%$ in MGC-803) and indicated that they were relatively insensitive to cetuximab (Figure 3A).

Next, to test whether RANKL modified the responsiveness of GC cell lines to cetuximab, the cells were preincubated with RANKL before addition of cetuximab. Compared with the treatment of cetuximab alone, the combination of RANKL and cetuximab significantly decreased inhibition of cell proliferation by cetuximab in SGC-7901 cells

Table 2 Spearman's correlations between EGFR and RANK expression in primary GC patients

\begin{tabular}{llllll}
\hline EGFR & Cases (\%) & \multicolumn{2}{l}{ RANK } & \multicolumn{3}{l}{ Spearman's r P-value } \\
\cline { 3 - 4 } & & Low (\%) & High (\%) & & \\
\hline Low (\%) & $49(72)$ & $33(67)$ & $16(33)$ & 0.510 & $<0.001$ \\
High (\%) & $19(28)$ & $2(10)$ & $17(90)$ & & \\
\hline
\end{tabular}

Abbreviation: GC, gastric cancer.
$(8.2 \% \pm 0.1 \%$ in the combined-treated arm vs $21.7 \% \pm 4.7 \%$ in the cetuximab-treatment arm, $P=0.009$ ) and MGC- 803 cells $(4.1 \% \pm 1.1 \%$ in the combined-treated arm vs $16.8 \% \pm 2.3 \%$ in the cetuximab-treatment arm, $P=0.0007$; Figure $3 \mathrm{~A}$ ). Furthermore, colony-forming assays showed that the combination of cetuximab and RANKL increased colony formation ability compared with cetuximab alone (Figure 3B).

We then explored the change in signaling when combined treated with cetuximab and RANKL in SGC-7901 cells. Cetuximab treatment alone mildly suppressed phosphorylation of EGFR, AKT and ERK, whereas combination of cetuximab and RANKL reversed this suppression (Figure 4).

These results showed that RANKL depressed sensitivity to cetuximab in GC cells through activation of EGFR and its downstream pathways.

\section{Blockade of RANKL/RANK pathway restores RANKL-induced cetuximab resistance in GC cells}

To further confirm the role of the RANKL/RANK pathway in sensitivity to cetuximab, we downregulated RANK by siRNA and then treated with cetuximab, RANKL, or a combination of them. MTT assay revealed that RANK knockdown resulted in reduction of cell proliferation by $\sim 20 \%$. Moreover, in the non-silencing (NS) control arm, cetuximab reduced proliferation by $17.7 \% \pm 4.4 \%$ and the combination 
A

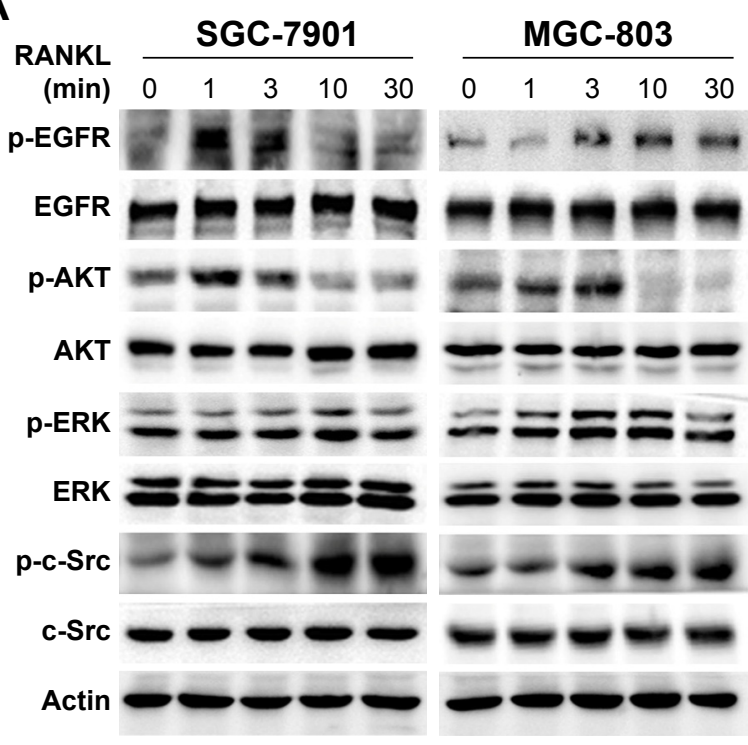

B
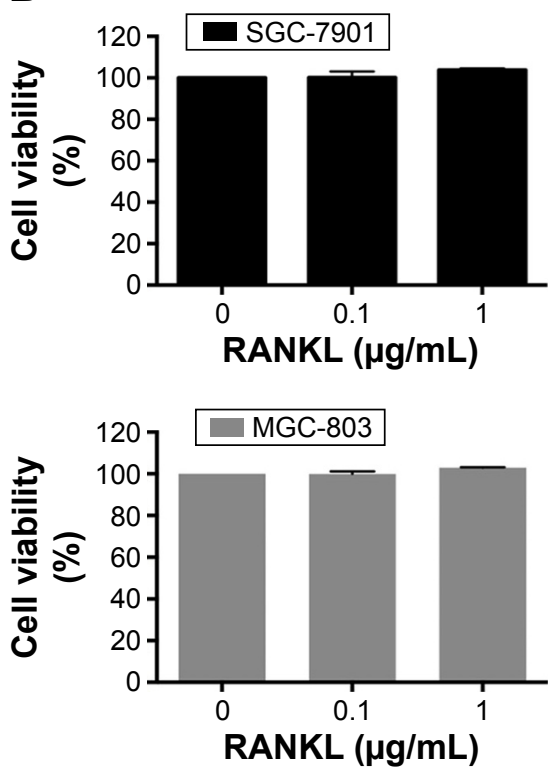

Figure 2 RANKL stimulated EGFR phosphorylation and downstream signaling in GC cells.

Notes: (A) Two RAS wide-type GC cell lines (SGC-790I and MGC-803) were treated with I $\mu \mathrm{g} / \mathrm{mL}$ sRANKL for the indicated times. The expression of EGFR, c-Src, AKT and ERK and phosphorylation levels were analyzed by Western blot. (B) SGC-790I and MGC-803 cells were respectively treated with sRANKL at $0.1 \mu g / \mathrm{mL}$ and I $\mu \mathrm{g} / \mathrm{mL}$ for 48 hours. Cell viability was assessed by MTT assay. The results from three independent experiments are shown.

Abbreviation: GC, gastric cancer.

of RANKL and cetuximab reduced proliferation by only $10.2 \% \pm 1.1 \%$. In the si-RANK arm, treatment with cetuximab inhibited proliferation by $45.1 \% \pm 4.8 \%$ compared with the non-treated control, while treatment combined with cetuximab and RANKL inhibited proliferation by $45.0 \% \pm 5.1 \%$. In the NS control arm, the inhibition rate of proliferation after cetuximab treatment was significantly higher than in the combination treatment, while there was no obvious change in the si-RANK arm (Figure 4A). Therefore, knockdown of RANK restored the RANKL-induced resistance to cetuximab in SGC-7901 cells. Furthermore, we used OPG that negatively regulates RANKL binding to RANK. As expected, in the control arm, the inhibition rate of proliferation in cetuximab-treated cells was significantly higher than that of the combined-treated cells, while there was no obvious change in the OPG arm. This indicated that inhibition of RANKL reduced RANKL-induced cetuximab resistance in SGC-7901 cells (Figure 4C). The results showed that both knockdown of RANK and inhibition of RANKL could reverse the effect of growth inhibition induced by cetuximab in SGC-7901 cells.

Changes in EGFR signaling by si-RANK or OPG in SGC-7901 cells were also determined. Compared to the NS control, reduced phosphorylation of EGFR, AKT and ERK was clearly detected when RANK was knocked down. Furthermore, RANK knockdown suppressed the effect of RANKL on EGFR, AKT and ERK activation (Figure 4B).
Similarly, OPG reversed RANKL-induced EGFR, AKT and ERK activation that mediated resistance to cetuximab (Figure 4D).

These results suggested that inhibition of the RANKL/ RANK pathway by RANK knockdown or RANKL inhibitor restored RANKL-induced cetuximab resistance through depression of EGFR, AKT and ERK phosphorylation.

\section{C-Src activation induced by RANKL is involved in resistance to cetuximab in GC cells}

We speculated that c-Src, an essential intermediate in the crosstalk between EGFR pathways and other tyrosine kinase receptors, might be involved in the interaction between EGFR and RANKL/RANK pathway. Activation of c-Src was previously observed when SGC-7901 and MGC-803 cells were stimulated with RANKL (Figure 2A). Then we used two selective c-Src kinase inhibitors, dasatinib and PP2, to examine the effect of c-Src on EGFR signaling in GC cells. The activation of c-Src, EGFR, AKT and ERK was dramatically attenuated in SGC-7901 and MGC-803 cells after treating with c-Src inhibitors (Figure 5A and B).

Next, we assessed the effect of c-Src on RANKLregulated cetuximab sensitivity. Dasatinib alone decreased the proliferation of SGC-7901 cells by $24.6 \% \pm 10.2 \%$. In the control arm, cetuximab treatment inhibited proliferation by 
A

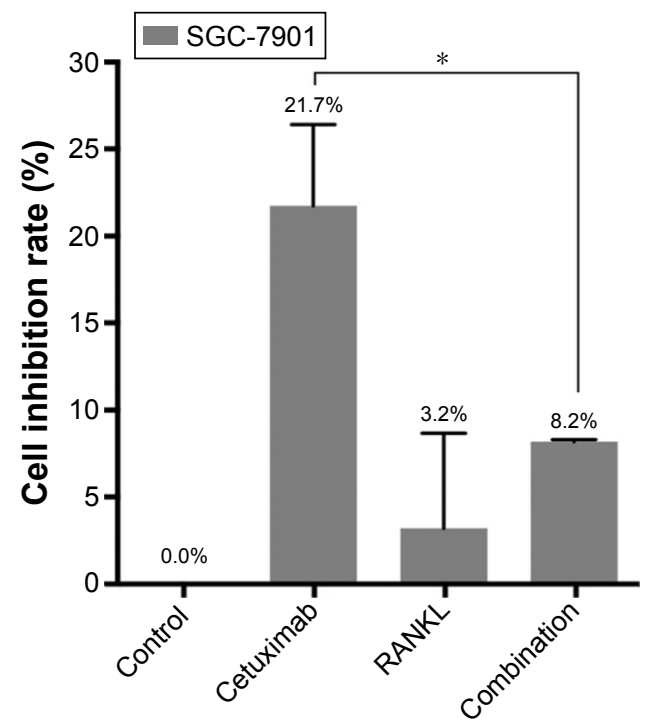

B

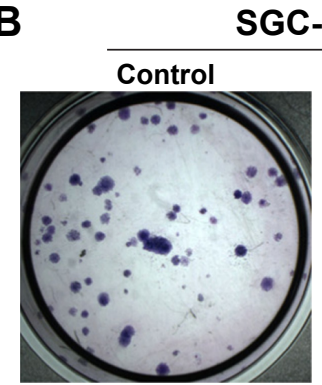

RANKL

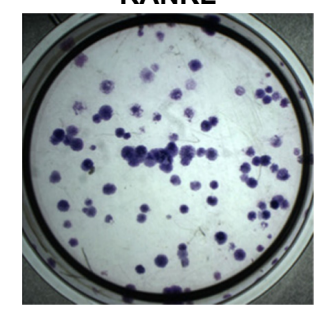

SGC-7901

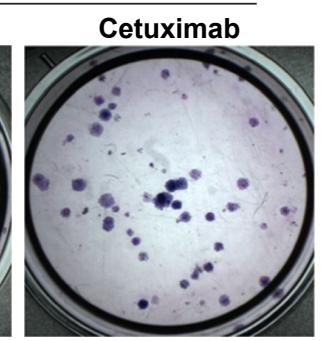

Combination

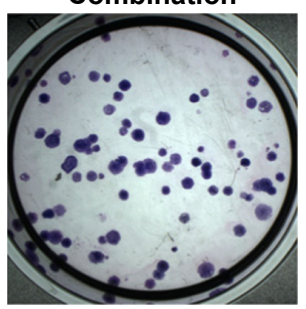

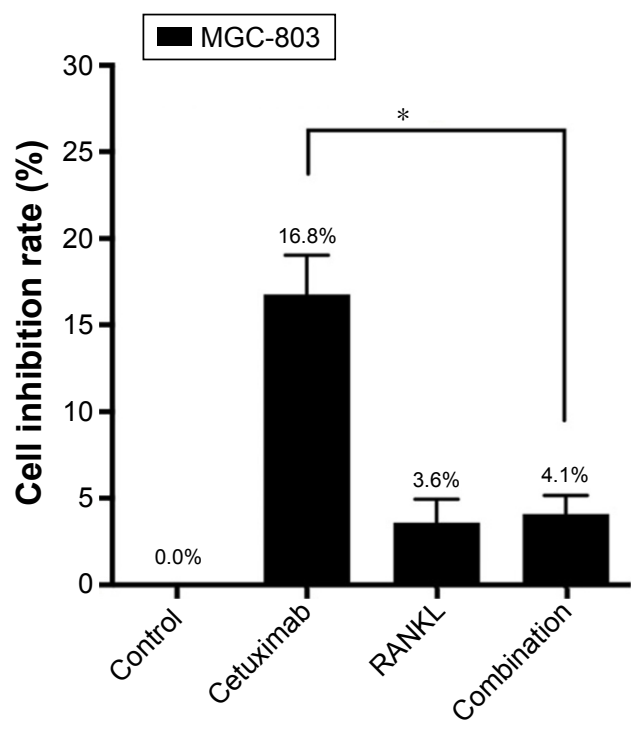

SGC-7901 MGC-803

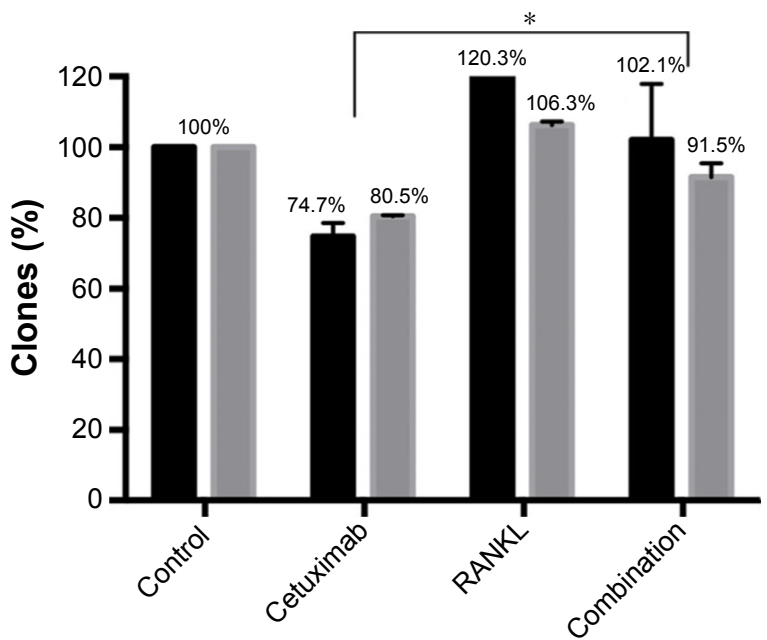

Figure 3 The effect of RANKL on sensitivity to cetuximab in GC cells.

Notes: (A) SGC-790I and MGC-803 cells were pretreated with I $\mu \mathrm{g} / \mathrm{mL}$ sRANKL for I hour and then incubated with $10 \mu \mathrm{g} / \mathrm{mL}$ cetuximab for 48 hours, followed by MTT assay. *Comparisons between the cetuximab-treated cells and the combined-treated cells, $P<0.05$. (B) SGC-790I and MGC-803 cells were pretreated with I $\mu$ g/mL sRANKL for I hour and then incubated with $10 \mu \mathrm{g} / \mathrm{mL}$ cetuximab for 14 days, and the clones were counted. ${ }^{*}$ Comparisons between the cetuximab-treated cells and the combined-treated cells, $P<0.05$.

Abbreviation: GC, gastric cancer.

$20.1 \% \pm 7.1 \%$, while the treatment combined with cetuximab and RANKL inhibited proliferation by $6.3 \% \pm 2.2 \%$. In the dasatinib arm, cetuximab treatment inhibited proliferation by $45.5 \% \pm 10.2 \%$, while the combination treatment inhibited proliferation by $46.4 \% \pm 10.4 \%$. In the control arm, the inhibitory effect of cetuximab on proliferation was much higher than that of the combination treatment, while no significant difference was found in the dasatinib arm. These results suggested that preincubated with dasatinib reversed RANKL-induced cetuximab resistance (Figure 5C).

Then, we examined the signaling change of EGFR signaling. Dasatinib not only resulted in the inhibition of EGFR,
AKT and ERK phosphorylation but also suppressed RANKLstimulated EGFR activation (Figure 5D). These results suggested that c-Src might be one of the key mediators in RANKL-induced EGFR activation that results in RANKLinduced cetuximab resistance.

\section{RANKL/RANK pathway also induces resistance to cetuximab in colon cancer cells}

We further explored whether RANKL/RANK pathway was involved in resistance to cetuximab in colon cancer cells. We chose Caco-2 cells with wild type of $R A S$ and $R A F$. 
A

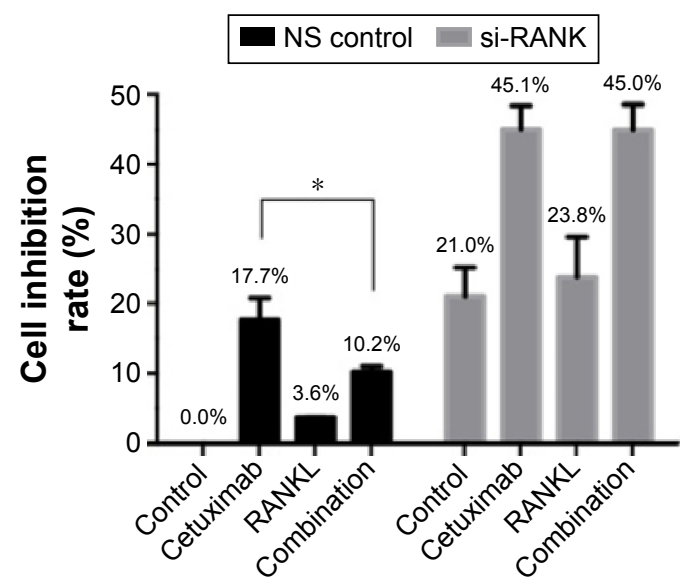

B

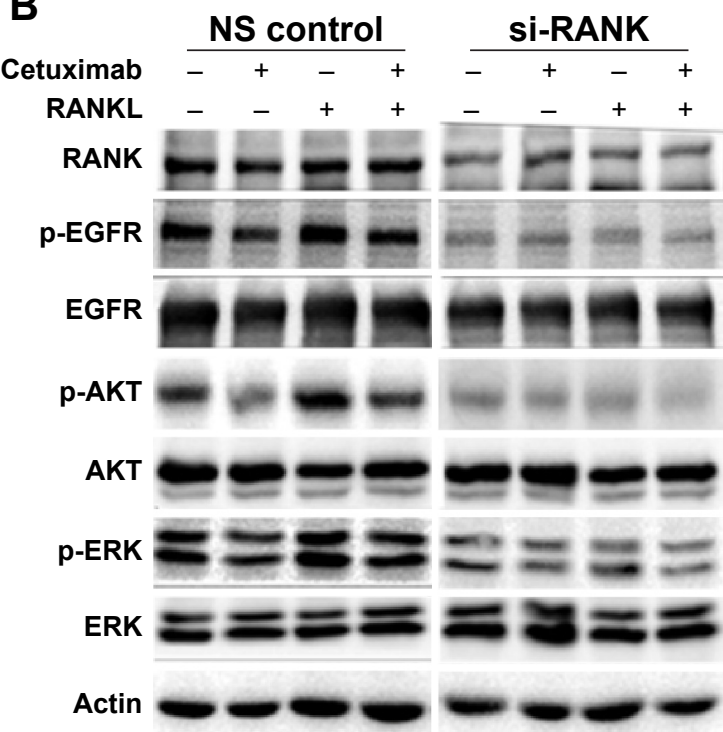

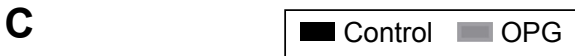

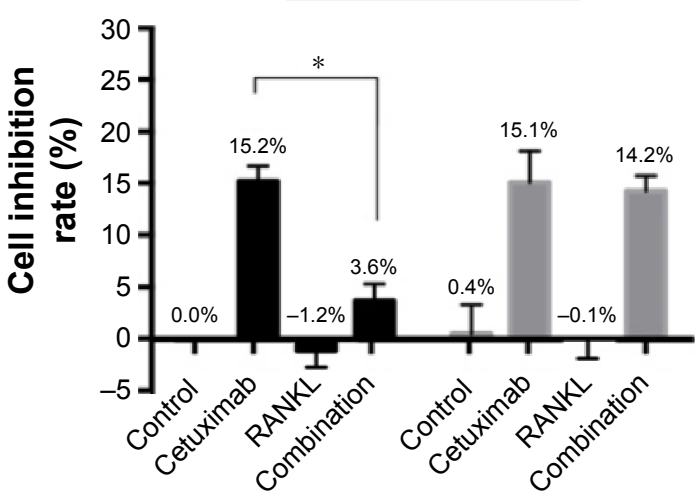

D

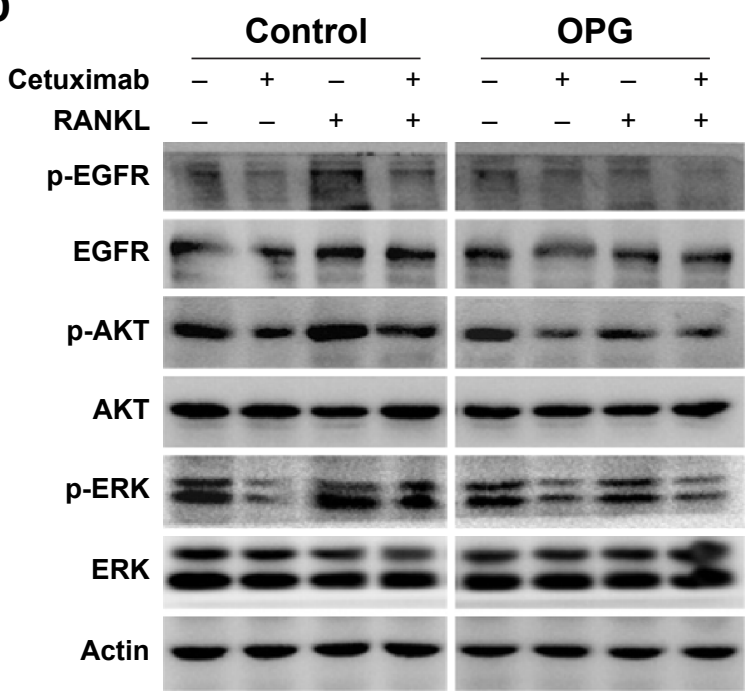

Figure 4 The effect of RANKL/RANK pathway on cetuximab sensitivity in SGC-790I cells.

Notes: (A) SGC-790I cells were transiently transfected with RANK siRNA for 24 hours and then pretreated with I $\mu \mathrm{g} / \mathrm{mL}$ sRANKL for I hour, followed by $10 \mu \mathrm{g} / \mathrm{mL}$ cetuximab for 48 hours. Cell viability was assessed by MTT assay. ${ }^{*}$ Comparisons between the cetuximab-treated cells and the combined-treated cells in the NS control arm, $P<0.05$. (B) After transient transfection with RANK siRNA for 48 hours, SGC-790I cells were pretreated with I $\mu g / \mathrm{mL} s R A N K L$ for I hour, followed by $10 \mu \mathrm{g} / \mathrm{mL}$ cetuximab for 48 hours. Expression of RANK, EGFR, AKT, ERK, and phosphorylation levels were analyzed by Western blot. (C) SGC-790I cells were preincubated with $10 \mu \mathrm{g} / \mathrm{mL}$ rOPG for 4 hours and then pretreated with I $\mu \mathrm{g} / \mathrm{mL}$ sRANKL for I hour, followed by $10 \mu \mathrm{g} / \mathrm{mL}$ cetuximab for 48 hours. Cell viability was assessed by MTT assay. *Comparisons between the cetuximab-treated cells and the combined-treated cells in the control arm, $P<0.05$. (D) After preincubating with $10 \mu g / m L ~ r O P G$ for 4 hours, SGC-790I cells were pretreated with I $\mu \mathrm{g} / \mathrm{mL}$ sRANKL for I hour, followed by $10 \mu \mathrm{g} / \mathrm{mL}$ cetuximab for 48 hours. Expression of EGFR, AKT, and ERK and phosphorylation levels were analyzed by Western blot.

Abbreviations: NS, non-silencing; siRNA, small interfering RNA.

Similarly, phosphorylation of EGFR, AKT, ERK, and c-Src was upregulated by RANKL (Figure 6A). Preincubation with RANKL also decreased sensitivity to cetuximab in Caco-2 cells (inhibition rate of $11.4 \% \pm 4.7 \%$ in the combined-treated arm vs $24.1 \% \pm 0.3 \%$ in the cetuximab-treatment arm, $P=0.01$; Figure 6B). Furthermore, downregulation of RANK with siRNA restored inhibition of cell proliferation by cetuximab (Figure 6C).

\section{Discussion}

EGFR served as one of the most relevant factors in the cell proliferation, survival, apoptosis, migration, and tumorigenesis of various cancers. Overexpression of EGFR has a significant predictive value for poor survival in GC patients. ${ }^{3}$ Subsequently, EGFR inhibition using cetuximab showed promise in combination with chemotherapy in several Phase II clinical studies for the treatment of metastatic GC. ${ }^{16-19}$ Unfortunately, no improvement in the overall survival was shown in facing the general population. ${ }^{5,6}$ Therefore, understanding the mechanisms of resistance to anti-EGFR treatments is of considerable significance and will help us to improve the response rate of anti-EGFR therapies. Among the various mechanisms of cetuximab resistance, aberrant activation of EGFR and downstream signaling pathways has been identified as a crucial resistance mechanism to anti-EGFR agents. In this study, we first demonstrated that EGFR expression 


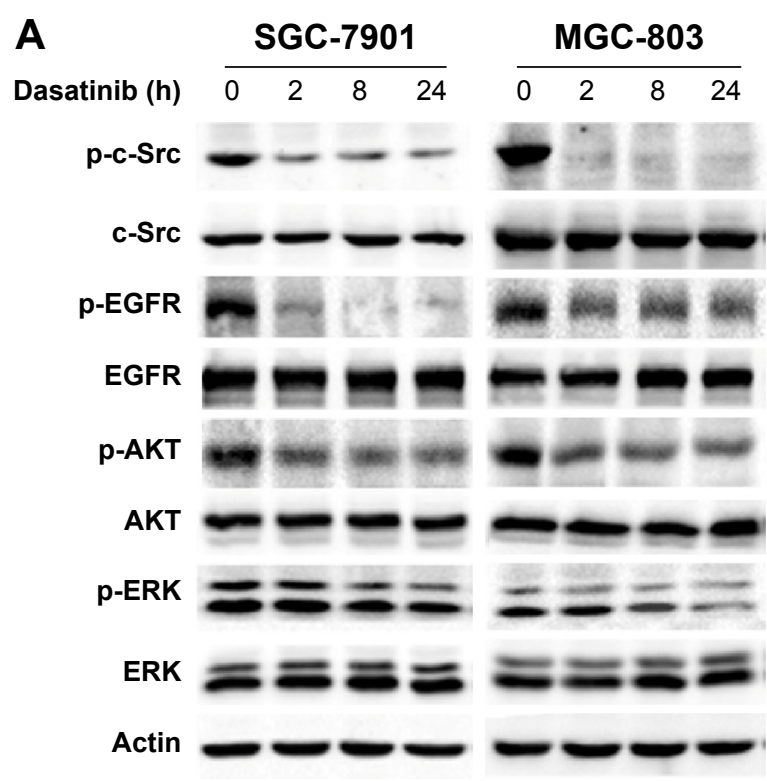

B

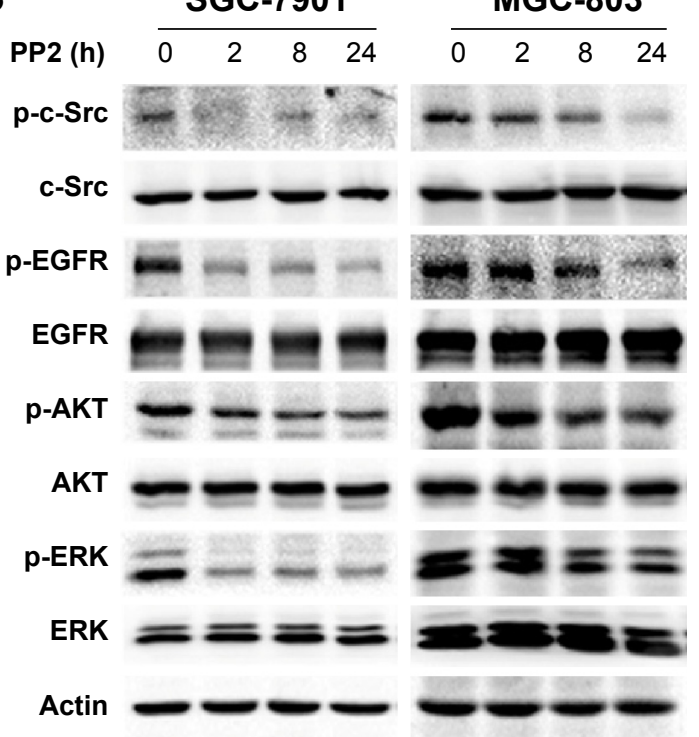

C

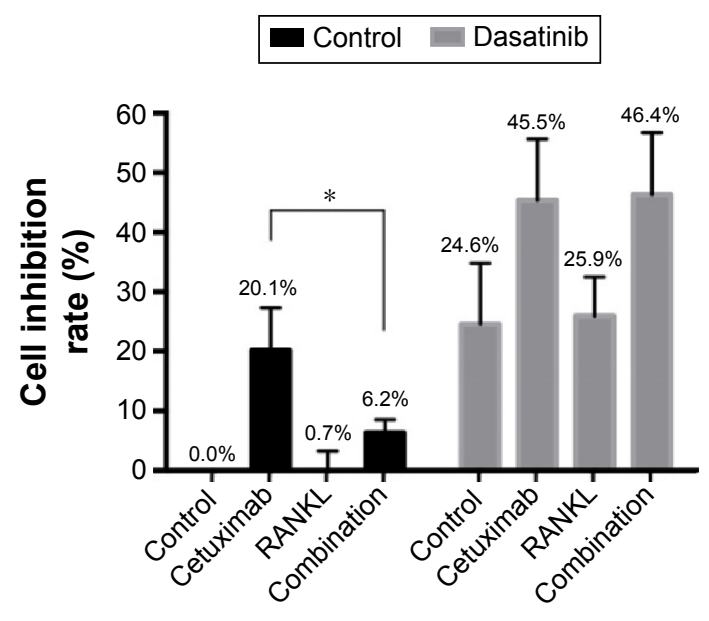

D

\section{Dasatinib}

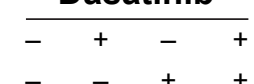

RANKL

p-c-Src

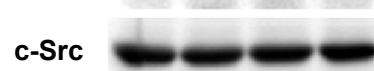

p-EGFR

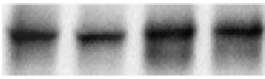

EGFR

p-AKT
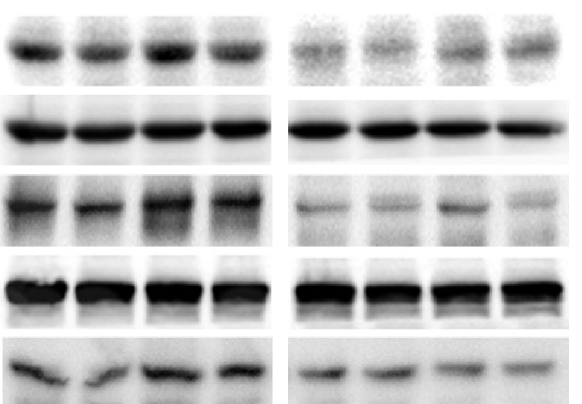

AKT

p-ERK

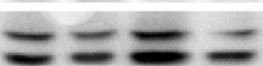

ERK

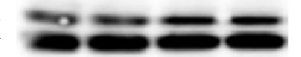

Actin

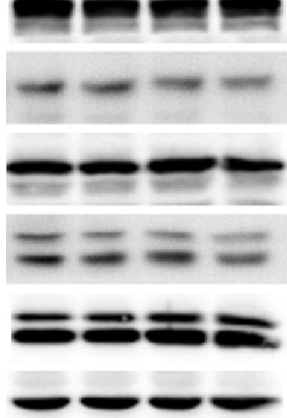

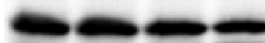

Figure 5 The effect of dasatinib on RANKL-induced cetuximab resistance in SGC-790 I cells.

Notes: (A and B) SGC-790I and MGC-803 cells were treated with $100 \mathrm{nM}$ dasatinib or $10 \mu \mathrm{M}$ PP2 for the indicated times. Expression of c-Src, EGFR, AKT, and ERK and phosphorylation levels were analyzed by Western blot. (C) SGC-790I cells were treated with $100 \mathrm{nM}$ dasatinib for 24 hours, followed by I $\mu \mathrm{g} / \mathrm{mL}$ sRANKL for I hour and then $10 \mu \mathrm{g} / \mathrm{mL}$ cetuximab for 48 hours. The cell viability was assessed by MTT assay. ${ }^{*}$ Comparisons between the cetuximab-treated cells and the combined-treated cells in the control arm, $P<0.05$. (D) SGC-790I cells were treated with or without $100 \mathrm{nM}$ dasatinib for 2 hours and then preincubated with I $\mu g / \mathrm{mL} s R A N K L$ for I hour, followed by $10 \mu \mathrm{g} / \mathrm{mL}$ cetuximab for 48 hours. The expression of c-Src, EGFR, AKT, and ERK and phosphorylation levels were analyzed by Western blot.

was positively correlated with RANK expression in GC patients, and RANKL activated EGFR signaling in GC cells that contributed to cetuximab resistance.

The RANKL/RANK pathway is associated with metastasis-related factors in several malignant tumors ${ }^{20-22}$ and hormone-induced tumorigenesis of breast cancer. ${ }^{23,24}$ The RANKL/RANK pathway has also been shown to regulate EGFR activation by cross talk during osteoclast differentiation. ${ }^{9}$ However, limited studies have focused on the relationship between the RANKL/RANK pathway and EGFR signaling in primary cancer cells. Our study originally explored the relationship between the RANKL/RANK pathway and EGFR in GC patients. Our IHC results showed that RANK and EGFR were highly expressed in GC tissues. 
A

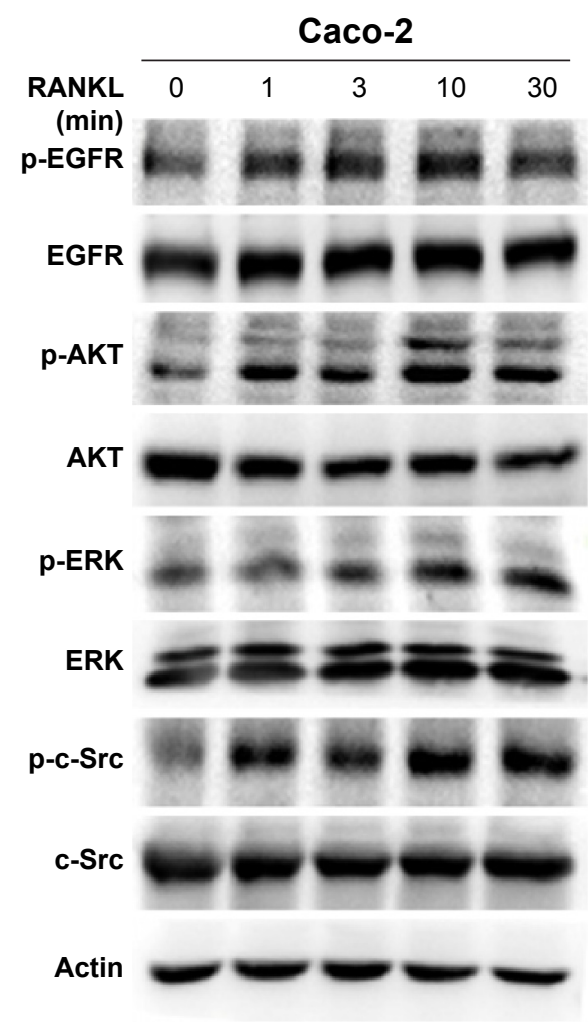

B
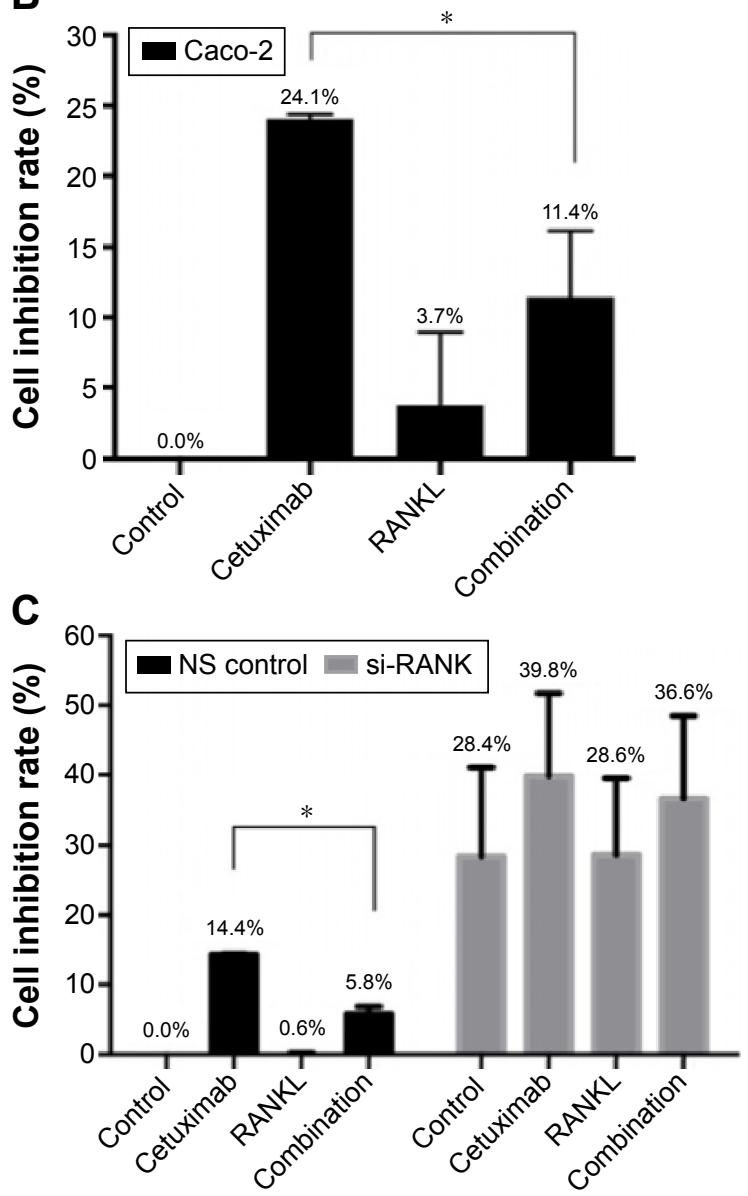

Figure 6 The effect of RANKL/RANK pathway on sensitivity to cetuximab in Caco-2 cells.

Notes: (A) Caco-2 cells were treated with I $\mu \mathrm{g} / \mathrm{mL}$ sRANKL for the indicated times. The expression of EGFR, AKT, ERK, and c-Src and phosphorylation levels were analyzed by Western blot. (B) Caco-2 cells were pretreated with I $\mu \mathrm{g} / \mathrm{mL}$ sRANKL for I hour and then incubated with $10 \mu \mathrm{g} / \mathrm{mL}$ cetuximab for 48 hours, followed by MTT assay. *Comparisons between cetuximab-treated arm and combined-treated arm, $P<0.05$. (C) Caco- 2 cells were transiently transfected with RANK siRNA for 24 hours and then pretreated with I $\mu \mathrm{g} / \mathrm{mL}$ sRANKL for I hour, followed by $10 \mu \mathrm{g} / \mathrm{mL}$ cetuximab for 48 hours. The cell viability was assessed by MTT assay. ${ }^{*}$ Comparisons between the cetuximab-treated cells and the combined-treated cells in the NS control arm, $P<0.05$.

Abbreviations: NS, non-silencing; siRNA, small interfering RNA.

We then showed that EGFR and RANK was expressed in several different GC cell lines (Figure 1B). In addition to this finding, we found a positive relationship between the expression of RANK and EGFR in GC tissues (Table 2). Furthermore, RANKL stimulation induced activation of EGFR and downstream signaling (Figure 2), which indicated the cross talk between the RANKL/RANK pathway and EGFR signaling.

Additionally, we observed that EGFR and downstream activation induced by RANKL decreased sensitivity to cetuximab in GC cells (Figures 2 and 3). Treatment with RANK siRNA or the RANKL inhibitor OPG attenuated phosphorylation of EGFR, AKT, and ERK and additionally reversed RANKL-induced resistance to cetuximab (Figure 4). Our results showed that the RANKL/RANK pathway phosphorylated EGFR-signaling cascade molecules, including EGFR itself, and further decreased sensitivity to cetuximab in GC cells. Furthermore, we investigated the role of the RANKL/RANK pathway after cetuximab treatment of colon cancer using the $R A S$ wild-type cell line Caco-2. We observed that sensitivity to cetuximab was similarly regulated by the RANKL/RANK pathway in this cell line (Figure 6). To our knowledge, this is the first evidence of a functional contribution of the RANKL/RANK pathway to cetuximab resistance that therefore makes it a promising target for developing novel therapeutic strategies to improve sensitivity to cetuximab.

To investigate the link between the RANKL/RANK pathway and EGFR signaling, we considered the role of c-Src. It is well established that c-Src and EGFR phosphorylate each other in a positive feedback loop. ${ }^{25,26}$ Our previous study showed that c-Src activation mediated cetuximab resistance 
in colon cancer cells, ${ }^{14}$ which have not been confirmed in GC yet. Moreover, our study showed that c-Src was activated by RANKL in the migration of breast cancer cells. ${ }^{13}$ Furthermore, c-Src expression showed a significantly positive linear relationship with RANK in breast cancer patients, ${ }^{27}$ indicating the relationship between c-Src and the RANKL/RANK pathway. In this study, we observed that c-Src was stimulated by RANKL (Figure 2A) and that c-Src inhibition led to repression of EGFR phosphorylation and its downstream factors in GC cells (Figure 5A and B). Consequently, inhibition of c-Src activity reversed RANKL-induced resistance to cetuximab (Figure 5C and D). These results suggested that c-Src is an intermediate between RANKL and EGFR and that c-Src activation contributes to cetuximab resistance via downregulation of EGFR signaling.

Our results showed that exogenous RANKL stimulated the RANKL/RANK pathway and then activated the EGFR pathway in GC cells. However, the accumulation of RANKL in gastric carcinoma remains elusive. The RANKL/RANK pathway plays an important role in the immune system, and RANKL expression is associated with $\mathrm{T}$ lymphocytes in breast cancer cells. ${ }^{28} \mathrm{GC}$ tissues were found infiltrated with T lymphocytes and associated with poor prognosis..$^{29}$ Herein, we initially speculated that $\mathrm{T}$ lymphocytes infiltrated into GC tissues would have high level of RANKL expression, and these might stimulate the RANKL/RANK pathway in the surrounding GC cells. As a result, the stimulated RANKL/RANK pathway might activate EGFR signaling and furthermore regulate the sensitivity of anti-EGFR therapies in GC.

Taken together, our results provided a potential mechanism for the resistance to anti-EGFR treatments in gastrointestinal tumors and a conclusion that RANKL/RANK and c-Src could be essential therapeutic targets to enhance sensitivity to anti-EGFR treatments.

\section{Acknowledgments}

This study was supported by Chinese National Foundation of National Sciences grants (No 81172198, No 81172369, No 81270036, No 81302128). Science and Technology Plan Project of Liaoning Province (No 2011404013-1 No 2014021089 No 2014225013). Liaoning BaiQianWan Talents Program (No 2014921032).

\section{Author contributions}

Xiujuan Qu and Yunpeng Liu conceived and designed the study. Xiaomeng Zhang, Na Song, Ye Zhang, Lingyun Zhang and Yan Wang performed the experiments. Yongxi Song and
Zhenning Wang provided the samples and collected patient information. Xiaomeng Zhang wrote the paper. Xiujuan Qu, Yunpeng Liu and Yongxi Song reviewed and edited the manuscript. All authors contributed toward data analysis, drafting and critically revising the paper and agree to be accountable for all aspects of the work.

\section{Disclosure}

The authors report no conflicts of interest in this work.

\section{References}

1. Torre LA, Bray F, Siegel RL, Ferlay J, Lortet-Tieulent J, Jemal A. Global cancer statistics, 2012. CA Cancer J Clin. 2015;65(2):87-108.

2. Wagner AD, Grothe W, Haerting J, Kleber G, Grothey A, Fleig WE. Chemotherapy in advanced gastric cancer: a systematic review and meta-analysis based on aggregate data. J Clin Oncol. 2006;24(18): 2903-2909.

3. Atmaca A, Werner D, Pauligk C, et al. The prognostic impact of epidermal growth factor receptor in patients with metastatic gastric cancer. BMC Cancer. 2012;12:524.

4. Kim JS, Kim MA, Kim TM, et al. Biomarker analysis in stage III-IV (M0) gastric cancer patients who received curative surgery followed by adjuvant 5 -fluorouracil and cisplatin chemotherapy: epidermal growth factor receptor (EGFR) associated with favourable survival. Br J Cancer. 2009;100(5):732-738.

5. Lordick F, Kang YK, Chung HC, et al. Capecitabine and cisplatin with or without cetuximab for patients with previously untreated advanced gastric cancer (EXPAND): a randomised, open-label phase 3 trial. Lancet Oncol. 2013;14(6):490-499.

6. Waddell T, Chau I, Cunningham D, et al. Epirubicin, oxaliplatin, and capecitabine with or without panitumumab for patients with previously untreated advanced oesophagogastric cancer (REAL3): a randomised, open-label phase 3 trial. Lancet Oncol. 2013;14(6):481-489.

7. Yano S, Wang W, Li Q, et al. Hepatocyte growth factor induces gefitinib resistance of lung adenocarcinoma with epidermal growth factor receptor-activating mutations. Cancer Res. 2008;68(22): 9479-9487.

8. Morgillo F, Kim WY, Kim ES, Ciardiello F, Hong WK, Lee HY. Implication of the insulin-like growth factor-IR pathway in the resistance of non-small cell lung cancer cells to treatment with gefitinib. Clin Cancer Res. 2007;13(9):2795-2803.

9. Yi T, Lee HL, Cha JH, et al. Epidermal growth factor receptor regulates osteoclast differentiation and survival through cross-talking with RANK signaling. J Cell Physiol. 2008;217(2):409-422.

10. Santini D, Perrone G, Roato I, et al. Expression pattern of receptor activator of NFkappaB (RANK) in a series of primary solid tumors and related bone metastases. J Cell Physiol. 2011;226(3):780-784.

11. Armstrong AP, Miller RE, Jones JC, Zhang J, Keller ET, Dougall WC. RANKL acts directly on RANK-expressing prostate tumor cells and mediates migration and expression of tumor metastasis genes. Prostate. 2008;68(1):92-104.

12. Jones DH, Nakashima T, Sanchez OH, et al. Regulation of cancer cell migration and bone metastasis by RANKL. Nature. 2006;440(7084): 692-696.

13. Zhang L, Teng Y, Zhang Y, et al. C-Src-mediated RANKL-induced breast cancer cell migration by activation of the ERK and Akt pathway. Oncol Lett. 2012;3(2):395-400.

14. Song N, Liu S, Zhang J, et al. Cetuximab-induced MET activation acts as a novel resistance mechanism in colon cancer cells. Int J Mol Sci. 2014; 15(4):5838-5851.

15. Li H, Xu L, Li C, et al. Ubiquitin ligase Cbl-b represses IGF-I-induced epithelial mesenchymal transition via ZEB2 and microRNA-200c regulation in gastric cancer cells. Mol Cancer. 2014;13:136. 
16. Lordick F, Luber B, Lorenzen S, et al. Cetuximab plus oxaliplatin/ leucovorin/5-fluorouracil in first-line metastatic gastric cancer: a phase II study of the Arbeitsgemeinschaft Internistische Onkologie (AIO). Br J Cancer. 2010;102(3):500-505.

17. Moehler M, Mueller A, Trarbach T, et al. Cetuximab with irinotecan, folinic acid and 5-fluorouracil as first-line treatment in advanced gastroesophageal cancer: a prospective multi-center biomarker-oriented phase II study. Ann Oncol. 2011;22(6):1358-1366.

18. Pinto C, Di Fabio F, Barone C, et al. Phase II study of cetuximab in combination with cisplatin and docetaxel in patients with untreated advanced gastric or gastro-oesophageal junction adenocarcinoma (DOCETUX study). Br J Cancer. 2009;101(8):1261-1268.

19. Pinto C, Di Fabio F, Siena S, et al. Phase II study of cetuximab in combination with FOLFIRI in patients with untreated advanced gastric or gastroesophageal junction adenocarcinoma (FOLCETUX study). Ann Oncol. 2007;18(3):510-517.

20. Tsubaki M, Komai M, Fujimoto S, et al. Activation of NF-kappaB by the RANKL/RANK system up-regulates snail and twist expressions and induces epithelial-to-mesenchymal transition in mammary tumor cell lines. J Exp Clin Cancer Res. 2013;32:62.

21. Wang J, Liu Y, Wang L, Sun X, Wang Y. Clinical prognostic significance and pro-metastatic activity of RANK/RANKL via the AKT pathway in endometrial cancer. Oncotarget. 2016;7(5):5564-5575.

22. Odero-Marah VA, Wang R, Chu G, et al. Receptor activator of NFkappaB Ligand (RANKL) expression is associated with epithelial to mesenchymal transition in human prostate cancer cells. Cell Res. 2008;18(8):858-870.
23. Schramek D, Leibbrandt A, Sigl V, et al. Osteoclast differentiation factor RANKL controls development of progestin-driven mammary cancer. Nature. 2010;468(7320):98-102.

24. Gonzalez-Suarez E, Jacob AP, Jones J, et al. RANK ligand mediates progestin-induced mammary epithelial proliferation and carcinogenesis. Nature. 2010;468(7320):103-107.

25. Biscardi JS, Maa MC, Tice DA, Cox ME, Leu TH, Parsons SJ. c-Srcmediated phosphorylation of the epidermal growth factor receptor on Tyr845 and Tyr1101 is associated with modulation of receptor function. J Biol Chem. 1999;274(12):8335-8343.

26. Stover DR, Becker M, Liebetanz J, Lydon NB. Src phosphorylation of the epidermal growth factor receptor at novel sites mediates receptor interaction with Src and P85 alpha. J Biol Chem. 1995;270(26):15591-15597.

27. Li R, Zhang K, Penedo TL, et al. The RANK pathway in advanced breast cancer: does Src play a role? Appl Immunohistochem Mol Morphol. 2016;24(1):42-50.

28. Tan W, Zhang W, Strasner A, et al. Fibroblast-recruited, tumorinfiltrating CD4+ T cells stimulate mammary cancer metastasis through RANKL-RANK signaling. Nature. 2011;470(7335):548-553.

29. Ohno S, Tachibana M, Fujii T, Ueda S, Kubota H, Nagasue N. Role of stromal collagen in immunomodulation and prognosis of advanced gastric carcinoma. Int J of Cancer. 2002;97(6):770-774.
OncoTargets and Therapy

\section{Publish your work in this journal}

OncoTargets and Therapy is an international, peer-reviewed, open access journal focusing on the pathological basis of all cancers, potential targets for therapy and treatment protocols employed to improve the management of cancer patients. The journal also focuses on the impact of management programs and new therapeutic agents and protocols on

\section{Dovepress}

patient perspectives such as quality of life, adherence and satisfaction. The manuscript management system is completely online and includes a very quick and fair peer-review system, which is all easy to use. Visit http://www.dovepress.com/testimonials.php to read real quotes from published authors. 